\title{
EXPERIMENTAL EVALUATION OF A DEBIASING METHOD FOR ANALYSIS IN ENGINEERING DESIGN
}

\author{
Nelius, Thomas; Matthiesen, Sven \\ Karlsruhe Institute of Technology (KIT)
}

\begin{abstract}
During analysis in engineering design, systematic thinking errors - so-called cognitive biases - can lead to inaccurate understanding of the design problem. With a simplified version of the Analysis of Competing Hypotheses - ACH method and a simplified decision matrix, the confirmation bias in particular can be minimized. To evaluate this method, it was taught to experienced design engineers and mechanical engineering students. During the experimental evaluation the participants analysed a real technical problem. The procedures and results were compared with a previously conducted study with the same task. The design engineers have not changed their approaches and could not further improve their analysis success. The students profited considerably from the training. They have mentioned twice as much supporting evidence and six times as much contradicting evidence through the training indicating a more extensive analysis. As a result, the students showed significantly fewer signs of confirmation bias than without training. The findings suggest that debiasing strategies should be introduced early in engineering design education.
\end{abstract}

Keywords: Decision making, Design cognition, Human behaviour in design, Confirmation Bias, Debiasing

Contact:

Matthiesen, Sven

Karlsruhe Institute of Technology (KIT)

Institute of Product Engineering

Germany

sven.matthiesen@kit.edu

Cite this article: Nelius, T., Matthiesen, S. (2019) 'Experimental Evaluation of a Debiasing Method for Analysis in Engineering Design', in Proceedings of the 22nd International Conference on Engineering Design (ICED19), Delft, The Netherlands, 5-8 August 2019. DOI:10.1017/dsi.2019.53 


\section{INTRODUCTION}

Many activities in engineering design are associated with problem solving and the entire design process can be understood as a problem solving process (Albers and Braun, 2011). A central step in problem solving is the identification of the cause of the problem. Only by a detailed understanding of the problem cause, the design engineer can develop a solution efficiently. The assumption of a wrong problem cause or a faulty understanding of the problem leads to time-consuming iterations in product development (Meboldt et al., 2012; Wynn and Eckert, 2017)This comprehension of the problem is built up in analysis phases in which the occurring system behaviour is compared to the desired function and associated with the physical embodiment. The correct analysis of the problem and its cause is therefore a central step in solving the problem. This paper therefore focuses on the correct analysis in engineering design. In order to develop a suitable support to avoid time-consuming iterations, the challenges and procedures for solving problems in engineering design must be understood.

Human behaviour has a substantial influence on problem solving. Especially systematic thinking errors, so-called cognitive bias, can have a negative influence on design engineers. The most studied cognitive bias is the confirmation bias. The confirmation bias describes the tendency to search and interpret evidence in such a way that it is consistent with existing beliefs. The confirmation bias is associated with the design fixation, which reinforces a fixation on initial ideas and the tendency to ignore contradictory information. (Hallihan et al., 2012)

We have therefore developed a debiasing method and investigated its usefulness for the analysis phases of engineering design.

The introduction is divided in three sections. First, the background of studies of analysis in engineering design is introduced. Secondly, the confirmation bias and debiasing approaches are presented. In the third section, own preliminary work is presented, in which challenges were identified during the analysis. We use the data from this preliminary work to experimentally evaluate the usefulness of the debiasing method, which is presented in chapter 2.1 .

\subsection{Analysis in engineering design}

Studies on the analysis in engineering design mostly focus on functional analysis - which is also known as functional decomposition. "Functional decomposition is a process that is typically used to assist engineers with identifying essential functions in various design tasks, including product dissection. It is a valuable tool used in industry to improve legacy products, understand competitor products, or help new employees learn about a company design." (Booth et al., 2015) Numerous studies have already been carried out on the functional analysis (Booth et al., 2015; Eckert et al., 2012; Hess et al., 2017; Ruckpaul et al., 2015). Usually, the study participants receive working devices that are unknown to them and are asked to identify their sub functions. These studies typically aim at identifying intuitive approaches or investigating given strategies such as top-down, energy-flow or enumeration.

A major part of the analysis in industrial product development is carried out on the company's own products. The function of the own products is known to the designer engineers, they are therefore more interested in the undesired system behaviour and its causes. The aim of the designers is to trace the undesired system behaviour back to certain design elements in order to be able to develop an improved development generation (Albers et al., 2017). The functional analysis does not depict this, since it only describes the identification of unknown functions of working systems (Matthiesen et al., 2017). Ruckpaul et al. (2014) speak therefore of synthesis-driven analysis in order to be able to describe the analysis of engineers during engineering design tasks.

Matthiesen et al. (2017) compared the functional analysis to the synthesis-driven analysis and have shown considerable differences in design engineers' approaches. For a realistic illustration of the analysis process, it is therefore necessary to give study participants a further development as a task and to observe the analysis during it. (Matthiesen et al., 2017)

In this study, we use a task representing a synthesis-driven analysis to better represent the challenges in real product development processes.

\subsection{Confirmation bias and debiasing}

When solving analysing problems, design engineers are subject to cognitive biases - systematic errors in human reasoning. The most common cognitive bias is the confirmation bias. Confirmation bias "connotes 
the seeking or interpreting of evidence in ways that are partial to existing beliefs, expectations, or a hypothesis in hand." (Nickerson 1998) The confirmation bias is probably the most prevalent and investigated cognitive bias, with research in psychology, medicine, law, software engineering and more. Confirmation bias can be classified into three error types: "interpretation errors where one misinterprets the meaning (directionality) of evidence to support one's preferred hypothesis, projection errors where one codes neutral evidence as supporting one's preferred hypothesis, and weighting errors where one correctly interprets evidence but assigns more or less importance to the evidence, depending on whether it supports or disconfirms a preferred hypothesis." (Lehner et al., 2008)

Since research on cognitive biases has been carried out, researchers have been looking for ways to overcome these biases. Strategies against confirmation biases are the establishment of several hypotheses, the search for evidence against assumptions and the parallel evaluation of hypotheses. The mere reference to the confirmation bias does not help to overcome it. This requires formal methods such as the Analysis of Competing Hypothesis - ACH, where the user has to set up several hypotheses and search for evidence for and against the hypothesis. To support the analysis a matrix is used, which matches the hypotheses and the evidence. (Heuer, 1999) Especially for inexperienced users, ACH can assist in the evaluation of evidence in intelligence analysis. (Lehner et al., 2008)

In the field of engineering design, cognitive biases are rarely investigated. Hallihan et al. (2012) have used a protocol study and demonstrated that confirmation bias is present during concept generation and concept evaluation. They also showed that decision matrices, based on the Analysis of Competing Hypothesis - ACH Method (Heuer, 1999) is an effective tool to reduce confirmation bias during concept evaluation. Participants should evaluate various concepts to test the hypothesis that designers are subject to design fixation (Hallihan et al., 2012). The known investigations, however, are limited to the early phase of product development. The tasks in existing studies usually represent only small aspects of the design. The investigations usually take place based on concepts on paper and there are no real systems or prototypes available. The particularly difficult technical problems, meanwhile, are in the late phase of product development (Smith and Tjandra, 1998).

We therefore see a strong need to investigate cognitive bias in the late phase of product development and to investigate appropriate debiasing strategies on realistic design tasks.

\subsection{Study on challenges and successful approaches during analysis}

The study presented in this paper (test study) is based on a previous study, referred to as main study. In the main study, we examined the approaches during analysis on a realistic engineering design task. Mechanical engineering students and experienced design engineers had to identify the cause for a broken part in a power tool. Students as well as experienced design engineers verified their assumptions by using mostly evidence that supports their own assumptions - indicating confirmation bias. This confirmation led to the problem that wrong assumptions and problem causes were assumed to be true. These participants then developed inappropriate solutions, which would have led to timeconsuming and expensive iterations in companies. Successful participants made multiple assumptions and verified their findings more often. Especially evidence against their assumptions improved the participants' understanding (Matthiesen and Nelius, 2018b) It can therefore be assumed that the confirmation bias occurs during analysis in engineering design. Successful participants have intuitively applied debiasing strategies.

\subsection{Objectives}

After having identified challenges by the confirmation bias and successful approaches in the preliminary work, we developed a method based on these findings and a further literature review to support the analysis of problem causes in engineering design. The training (see chapter 2.1) covers thinking errors during analysis and successful strategies. This includes a simplified version of the $\mathrm{ACH}$ decision matrix. In this paper, we will examine how the training supports the analysis of technical systems in the late product development phase.

\section{EXPERIMENTAL DESIGN}

This paper is based on a previously conducted main study. In this main study, we identified successful approaches and challenges in synthesis-driven analysis (Matthiesen and Nelius, 2018b). In order to make use of these findings, we have developed a training for the analysis. Students and experienced 
design engineers were trained in this test study and solve the task of the main study. We compare the test study with the main study (chapter 1.3) to evaluate the benefit of the training.

\subsection{Procedure}

The test study consists of three parts for the participants. (1.) A theoretical training session for the debiasing method, (2.) practice of the debiasing method in a hands-on training and (3.) the task from the main study. The training took place in small groups of 3-6 participants. The participants completed the task individually.

\section{Theoretical Training Session}

The participants were briefed on the challenges of analysing technical systems during the training. Frequent errors and confirmation bias that can occur during the analysis were addressed. For example, that participants in the main study often tracked wrong causes of problems and did not notice this.

In addition, the approaches with which the successful participants have overcome these challenges were described (Setting up several hypotheses, frequent checking of one's own understanding of the system, focus on evidence which contradicts the favoured hypothesis). In order to reduce the confirmation bias, the participants were trained to formulate many hypotheses. In addition, the participants should focus their analysis on finding counter-indications for their hypotheses so that they can reject false hypotheses.

In order to support these approaches, the participants have been taught a simplified version of the Analysis of Competing Hypothesis method from Heuer (1999). Instead of eight steps, we presented a four-step approach. (1.) The participants should formulate the problem as precisely as possible in order to focus their analysis. (2.) They should identify several possible causes of the problem and write them down in the columns of the decision matrix. (3.) The rows of the decision matrix should be filled with supporting evidence and especially contradicting evidence. Steps 2 and 3 of the method can be repeated iteratively, since new evidence and causes can constantly be identified during the analysis. (4.) Finally, a conscious selection of the most probable cause should take place or it should be indicated that the cause has not yet been identified.

\section{Hands-On Training}

The approach was practiced on another system to get the participants to know the procedure and to ensure the correct application of the matrix. For this purpose a further task was set in which the procedure could be practiced. The task was to find a malfunction in a power tool. A function of the system is only insufficiently fulfilled due to a tolerance problem. To give the test group no advantage, the problems differ considerably - a tolerance problem in the hands-on training and a component failure in the test study. The participants got a malfunctioning system alone or in pairs (depending on group size) and should find the problem cause. The trainer was available for questions and made sure that the decision matrix was used correctly. A completed decision matrix from the hands-on training is shown in the appendix. The bold contents were given. The contents in italics were filled in by a participant.

\section{Task: Synthesis-Driven Analysis}

For comparability, the task from the main study (Matthiesen and Nelius, 2018b) was used again, see Figure 1.

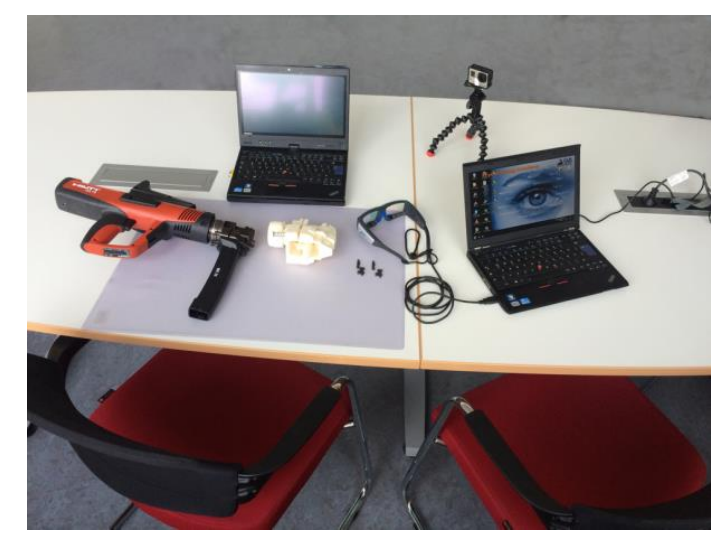

Figure 1. Experimental setup - synthesis-driven analysis (Matthiesen and Nelius, 2018a) 
The participants received a PowerPoint presentation with the task and information on the system. Thus, the influence of the moderator was reduced. The task was based on a real problem from a company.

In the late prototype phase, a safety-relevant part of a power tool failed. The task for the participants was a redesign to prevent the failure. This placed the analysis in a realistic context. The participants spent most of their time analysing the problem. The redesign was limited to simple sketches of possible alternatives. No complex dimensioning or detailed designs were necessary. The participants should first analyse the cause of the problem. For this, they got the prototype, the broken parts with wear marks, the technical drawings and an enlarged model. The participants could finish the task when they had solved the problem, after 20 minutes the moderator finished the task.

\subsection{Data acquisition and analysis}

Data Acquisition and Analysis is conducted as in the main study (Matthiesen and Nelius, 2018b) in order to compare the data with each other. The participants should use concurrent think aloud where the subjects speak their thoughts out loud during the task (Ericsson and Simon, 1993). This makes it possible to observe their analysis and argumentation during the task. The concurrent think aloud was practiced before the task and during the task the participants were reminded if they had not spoken for a longer time. The recorded data was examined on how often a participant verified an assumption during their analysis in which they have re-examined an issue - which will be referred to as verification. The verifications of assumptions during the analysis were additionally coded on the correctness of the participants' understanding before and after the verification. The verifications were categorised according to whether the information was in support of or against the assumption. A second coder reviewed all found verifications. When there were discrepancies between the two coders they discussed them and acquired consent.

Analysis success was determined by a questionnaire on the systems behaviour handed out after the analysis. The participants could get 9 points for correct answers on the system's behaviour. The analysis success is statistically processed by means of a Mann-Whitney-U-Test. The questionnaire also asked about the usefulness of the matrix.

The filled-in matrices were also analysed and the number of assumptions, supporting evidence and contradicted evidence were counted.

\subsection{Participants}

13 participants took part in the test study. As in the main study, mechanical engineering students and experienced design engineers participated in the test study. There were 7 students who were between their 5th and 12th semester, including undergraduate and graduate students. The prerequisite for participation in the study for the students was the successful completion of the Mechanical Design course (4 semesters, 20 ECTS, including lectures, tutorials and design workshops). Among the participants were 6 male and 1 female students. The 6 design engineers, all male, had between 1 and 30 years of work experience (14 years on average). One of the designers could not be evaluated because he did not use concurrent think aloud as instructed and therefore it is unclear how he proceeded with the analysis. Therefore, only the data of the remaining 12 test persons ( 7 students, 5 designers) were evaluated.

\section{RESULTS}

The participants worked with the decision matrix and filled it in during the task (example in the appendix). The students noted on average 3.3 assumptions $(\mathrm{sd}=0.8), 1.7$ supporting evidence ( $\mathrm{sd}=$ $1.6)$ and 1 contradicting evidence $(\mathrm{sd}=0.8)$. The design engineers noted on average 2.6 assumptions $(\mathrm{sd}=0.9), 1.4$ supporting evidence $(\mathrm{sd}=0.9)$ and 1 contradicting evidence $(\mathrm{sd}=0.7) .5$ of the 12 participants have recognized that they have not identified the correct causes of the problem. In the main study, only 2 out of 22 participants recognized that they had not identified the correct cause.

The participants have not entered all their assumptions and gathered information into the matrix, therefore the think aloud was considered additionally. Table 1 shows the evaluation of the think aloud during the task compared to the same task in the main study without training and matrix. 
Table 1. Number of verifications in the think aloud protocol grouped by evidence for/against the assumption (per participant) and analysis success

\begin{tabular}{|l|ccc|ccc|}
\hline & \multicolumn{3}{|c|}{ Students } & \multicolumn{3}{c|}{ Design Engineers } \\
\cline { 2 - 7 } & $\begin{array}{c}\text { Evidence } \\
\text { for the } \\
\text { Assumption }\end{array}$ & $\begin{array}{c}\text { Evidence } \\
\text { against the } \\
\text { Assumption }\end{array}$ & $\begin{array}{c}\text { Analysis } \\
\text { Success }\end{array}$ & $\begin{array}{c}\text { Evidence } \\
\text { for the } \\
\text { Assumption }\end{array}$ & $\begin{array}{c}\text { Evidence } \\
\text { against the } \\
\text { Assumption }\end{array}$ & $\begin{array}{c}\text { Analysis } \\
\text { Success }\end{array}$ \\
\hline Main Study & & & & & & \\
Mean & 0.92 & 0.38 & $37.5 \%$ & 1.73 & 0.36 & $58.4 \%$ \\
Standard Deviation & 1.04 & 0.51 & $15.3 \%$ & 1.42 & 0.50 & $0.25 \%$ \\
Test Study & & & & & & \\
Mean & 1.86 & 2.43 & $48.9 \%$ & 1.60 & 0.40 & $54.8 \%$ \\
Standard Deviation & 0.9 & 1.27 & $15.9 \%$ & 1.67 & 0.55 & $21.2 \%$ \\
\hline
\end{tabular}

The number of verifications increased among the students, who found evidence against their assumptions much more frequently (Verification with evidence against the assumption: 0.38 in the main study 2.43 in the test study).

The students' success in analysis increased significantly (mean $=37.5 \%$ in the main study | mean $=48.9 \%$ in the test study), exact Mann-Whitney-U-Test: $\mathrm{U}=22.5, \mathrm{p}=.036$. The effect strength is $\mathrm{r}=.41$ and corresponds to a medium effect.

The number of verifications remained the same for the design engineers. The success of the analysis also remained the same for the design engineers (main study: $58.4 \%$ |test study: $54.8 \%$ ).

For each verification, it was analysed whether it improved, did not change or even worsened the understanding of the participants. In addition, the verifications were classified as to whether evidence for or against the assumption was found. Table 2 shows the results of this analysis. 24 out of 58 verifications involved evidence against the assumption. This ratio is substantially higher than in the main study (16 out of 64 verifications with evidence against the assumption). Especially the verifications with evidence against their assumption improved the participants' understanding of the system. No verification resulted in a decrease in the participants understanding.

Table 2. Classifications of the verifications

\begin{tabular}{|l|c|c|c|}
\hline \multirow{2}{*}{} & \multirow{2}{*}{\begin{tabular}{c} 
Total \\
Number of \\
\cline { 3 - 4 }
\end{tabular}} & \multicolumn{2}{|c|}{ Classification in } \\
\cline { 3 - 4 } & Evidence for the & $\begin{array}{c}\text { Evidence } \\
\text { against the } \\
\text { Assumptions }\end{array}$ \\
\hline Improvements & 24 & 2 & 22 \\
\hline No Change & 34 & 32 & 2 \\
\hline Worsening & 0 & 0 & 0 \\
\hline & & 34 & 24 \\
\hline Total & 58 & \multicolumn{2}{|c|}{58} \\
\hline
\end{tabular}

\section{Qualitative evaluation of the matrix by the participants}

At the end of the task, the participants assessed the impact of the decision matrix on a scale of 1 (not useful) - 7 (very useful). The students rated the impact of the decision matrix lower (mean $=4.9$, $\mathrm{sd}=1.4$ ) than the design engineers (mean $=5.8, \mathrm{sd}=1.1$ ).

During a final discussion, the participants complained that filling in the matrix took time, which they lacked to complete the rest of the task. In addition, some participants found it difficult to formulate their evidence. On the other hand, some participants appreciated explicating their evidence as it forced them to think the reasoning through more deeply. The participants rated the matrix positively, as it leads them to make several assumptions and focused on the evidence against their presumption. 


\section{DIscussion}

To evaluate the method and the decision matrix, we consider the criteria applicability and usefulness of the decision matrix as recommended by Blessing and Chakrabarti (2009).

\subsection{Applicability of the debiasing method}

Based on the filled matrices, it can be observed that both groups of participants used the matrix in the test study similarly frequent (students: 3.3 assumptions, 2.7 evidence | design engineers: 2.6 assumptions, 2.4 evidence). However, the think aloud protocols show that the students reviewed their assumptions considerable more frequently. Through the training and the matrix, the students have more verifications (test study: 4.3 verifications - main study: 1.3 verifications) than the design engineers (test study: 2.0 verifications - main study: 2.1 verifications). Even though the students did not note all their verifications in the matrix, they have focused more intensively on them.

In the main study, students used less evidence for their analysis and focused on evidence in favour of their assumptions. Through training and the decision matrix, they used twice as much evidence for and six times more evidence against their assumptions. The design engineers showed no distinct change in their number of evidences (see Table 1).

\subsection{Usefulness of the debiasing method}

Due to the training and the matrix, the students performed considerably better than the control group in the main study. The design engineers achieved no additional improvement through training and the matrix.

We see two possible explanations for this:

- The design engineers have not changed their approach through the training. They have the similar number of verifications as in the control group. These results coincide with findings from Lehner et al. (2008), which show that inexperienced users benefit from debiasing methods while experienced users remain equally successful. The experienced designers had the same analytical success in the main study and test study, which can be explained by the lack of application of the debiasing strategy. The long practiced procedures of the experienced participants seem to be difficult to change by a short training, which would require longer training sessions or continuous coaching in the everyday work.

- The design engineers have already achieved very good results in the main study, which makes further improvements difficult to achieve. Lehner et al. (2008) also describe that experienced intelligence analysts are less prone to confirmation bias. This effect can be investigated with additional and more difficult tasks.

The decision matrix has supported the participants to recognize themselves that they have not yet identified the correct cause. In the main study, this ratio was considerably lower. As a result, solutions were developed for problem causes that were not present. The fact that the matrix contributed to this improved self-assessment can be seen as a notable success. Because wrong assumptions often lead to long iterations in product development (Meboldt et al., 2012; Wynn and Eckert, 2017).

The training has led to a significant improvement for the students while having no visible effect on the experienced design engineers. This contrasts with the subjective evaluation of the usefulness of the training. The design engineers rated the training more useful than the students did. The students thus benefited more from the decision matrix, but did not notice this. Therefore, when evaluating methods, both the perceived benefit for the user should be evaluated as well as the actual benefit should be assessed in an experiment.

\subsection{Limitations}

Due to the small sample of 12 test persons, it was not possible to determine the statistical significance of the results.

By using think aloud, the procedures of the subjects can be influenced. The scientific community has different views about this. Especially in the investigation of cognitive processes, think aloud can lead to undesired influences. According to Rozenblit and Keil (2002), the explanation of mechanical systems reduces the own overestimation and therefore confirmation bias. On the other hand, pronunciation can lead to a greater commitment to one's own position. The greater the commitment to 
a hypothesis, the more uncomfortable it is to have to give up the hypothesis again, and the greater could be the unconscious tendency to do anything to avoid it (Schweizer, 2005). Think aloud could therefore influence the confirmation bias in different directions. This would have to be investigated in further studies.

\subsection{Implications for education}

In order to further reduce the effects of the confirmation bias, we suggest the following training aspects.

- $\quad$ Training with more practice tasks

In this study, participants had only one exercise task with the decision matrix before their performance was evaluated. The exercise with several tasks should make the method easier to use and further increase the benefit.

- $\quad$ Direct Feedback and Reflection

When working on such training tasks, direct feedback should be given to the user. Was the method used correctly? Was the evidence interpreted correctly? Was the correct cause of the problem found? With this feedback, the user can improve and internalize his approach through reflection.

- Practice of debiasing methods in academic education

Debiasing methods such as ACH should be taught engineering students. These methods seem to be very complex and time-consuming, and therefore not suitable for everyday work. But the early practice of these methods without time pressure makes the application of the principles and the critical way of thinking possible to use under time pressure.

\section{CONCLUSION}

This paper builds on the previous main study. This main study $(\mathrm{n}=24)$ examined challenges and successful approaches in engineering design analysis. It has been shown that the confirmation bias can lead to the investigation of incorrect causes of problems. Successful participants prevented this by verifying their findings more often. They used evidence against their assumptions to falsify them particularly often. Based on the findings of the main study, a method was developed, which was presented and evaluated in this paper. 12 further participants (experienced design engineers and students) received a theoretical and a hands-on training session, which covered the application of the debiasing method and the decision matrix. The students clearly benefited from the training. The experienced design engineers did not show changes in their approach and the success of the analysis. The early practice of these procedures is therefore necessary because, as shown, changes with increasing experience are difficult to achieve. Strategies and methods to reduce cognitive biases should therefore be introduced more into the education of engineering design. This will allow future design engineers to practice and incorporate these successful approaches at an early stage of their qualification.

\section{REFERENCES}

Albers, A. and Braun, A. (2011), “A generalised framework to compass and to support complex product engineering processes". International Journal of Product Development, Vol. 15 No. 1-3, pp. 6-25.

Albers, A., Bursac, N. and Rapp, S. (2017), "PGE-Produktgenerationsentwicklung am Beispiel des Zweimassenschwungrads". Forschung im Ingenieurwesen, Vol. 81 No. 1, pp. 13-31.

Blessing, L. T. and Chakrabarti, A. (2009), “DRM, a design research methodology”. Springer Science \& Business Media.

Booth, J. W., Reid, T. N., Eckert, C. and Ramani, K. (2015), "Comparing functional analysis methods for product dissection tasks". Journal of Mechanical Design, Vol. 137 No. 8.

Eckert, C., Ruckpaul, A., Alink, T. and Albers, A. (2012), "Variations in functional decomposition for an existing product: Experimental results". AI EDAM, Vol. 26 No. 2, pp. 107-128.

Ericsson, K. A. and Simon, H. A. (1993), Protocol analysis. Cambridge, MA: MIT press.

Hallihan, G. M., Cheong, H. and Shu, L. H. (2012, August), "Confirmation and cognitive bias in design cognition". In ASME 2012 International Design Engineering Technical Conferences and Computers and Information in Engineering Conference (pp. 913-924). American Society of Mechanical Engineers.

Hess, S., Lohmeyer, Q. and Meboldt, M. (2017), "Utilization of Mobile Eye Tracking Data to Improve Engineering Design Education". In DS 88: Proceedings of the 19th International Conference on Engineering and Product 
Design Education (E\&PDE17), Building Community: Design Education for a Sustainable Future, Oslo, Norway, 7 \& 8 September 2017 (pp. 002-007).

Heuer, R. J. (1999), Psychology of intelligence analysis. United States Govt Printing Office

Lehner, P. E., Adelman, L., Cheikes, B. A. and Brown, M. J. (2008), "Confirmation bias in complex analyses". IEEE Transactions on Systems, Man, and Cybernetics-Part A: Systems and Humans, Vol. 38 No. 3, pp. 584592.

Matthiesen, S. and Nelius, T. (2018a). "Eye Tracking Study On Successful Micro-Strategies By Design Engineers For The Synthesis-Driven Analysis Of Technical Systems”. In Proceedings of TMCE 2018, Las Palmas de Gran Canaria, Spain, 7-11 May, 2018

Matthiesen, S. and Nelius, T. (2018b). "Managing Assumptions during Analysis - Study on successful Approaches of Design Engineers". DS 91: Proceedings of NordDesign 2018, Linköping, Sweden, 14th-17th August 2018.

Matthiesen, S., Nelius, T., Pflegler, B. and Gutmann, T. (2017), "Studiendesign zur Untersuchung der synthesegetriebenen Analyse von Konstrukteuren”. In DFX 2017: Proceedings of the 28th Symposium Design for X, 4-5 October 2017, Bamberg, Germany (pp. 299-310).

Meboldt, M., Matthiesen, S. and Lohmeyer, Q. (2012), "The dilemma of managing iterations in time-to-market development processes". In Second International Workshop on the Modelling and Management of Engineering Processes (MMEP 2012). Eidgenössische Technische Hochschule Zürich.

Nickerson, R. S. (1998), “Confirmation bias: A ubiquitous phenomenon in many guises". Review of general psychology, Vol. 2 No. 2, pp. 175.

Rozenblit, L. and Keil, F. (2002), "The misunderstood limits of folk science: An illusion of explanatory depth". Cognitive science, Vol. 26 No. 5, pp. 521-562.

Ruckpaul, A., Kriltz, A. and Matthiesen, S. (2014), 'Using eye tracking to understand the engineering designers' behavior in synthesis-driven analyzing processes: experiences in study design”. In International conference on human behavior in design HBiD. Proceedings of International conference on Human Behavior in Design, Ascona, Switzerland, 14-17 October, 2014

Ruckpaul, A., Nelius, T. and Matthiesen, S. (2015), "Differences in analysis and interpretation of technical systems by expert and novice engineering designers". In DS 80-2 Proceedings of the 20th International Conference on Engineering Design (ICED 15) Vol 2: Design Theory and Research Methodology Design Processes, Milan, Italy, 27-30.07.15

Schweizer, M. (2005), Kognitive Täuschungen vor Gericht. Eine empirische Studie. Dissertation, Rechtswissenschaftlichen Fakultät der Universität Zürich

Smith, R. P. and Tjandra, P. (1998), "Experimental observation of iteration in engineering design". Research in Engineering Design, Vol. 10 No. 2, pp. 107-117.

Wynn, D. C. and Eckert, C. M. (2017), "Perspectives on iteration in design and development". Research in Engineering Design, Vol. 28 No. 2, pp. 153-184.

\section{ACKNOWLEDGMENTS}

We would like to thank the German Research Foundation for funding and therefore enabling this research project "MA 5940/3-1 - Development of a methodical approach for generating embodiment function-correlations for engineers based on the examination of the courses of action during the design process". Further, we thank all the participants who took part in this study. 
Table 3. Exemplary decision matrix from the hands-on training

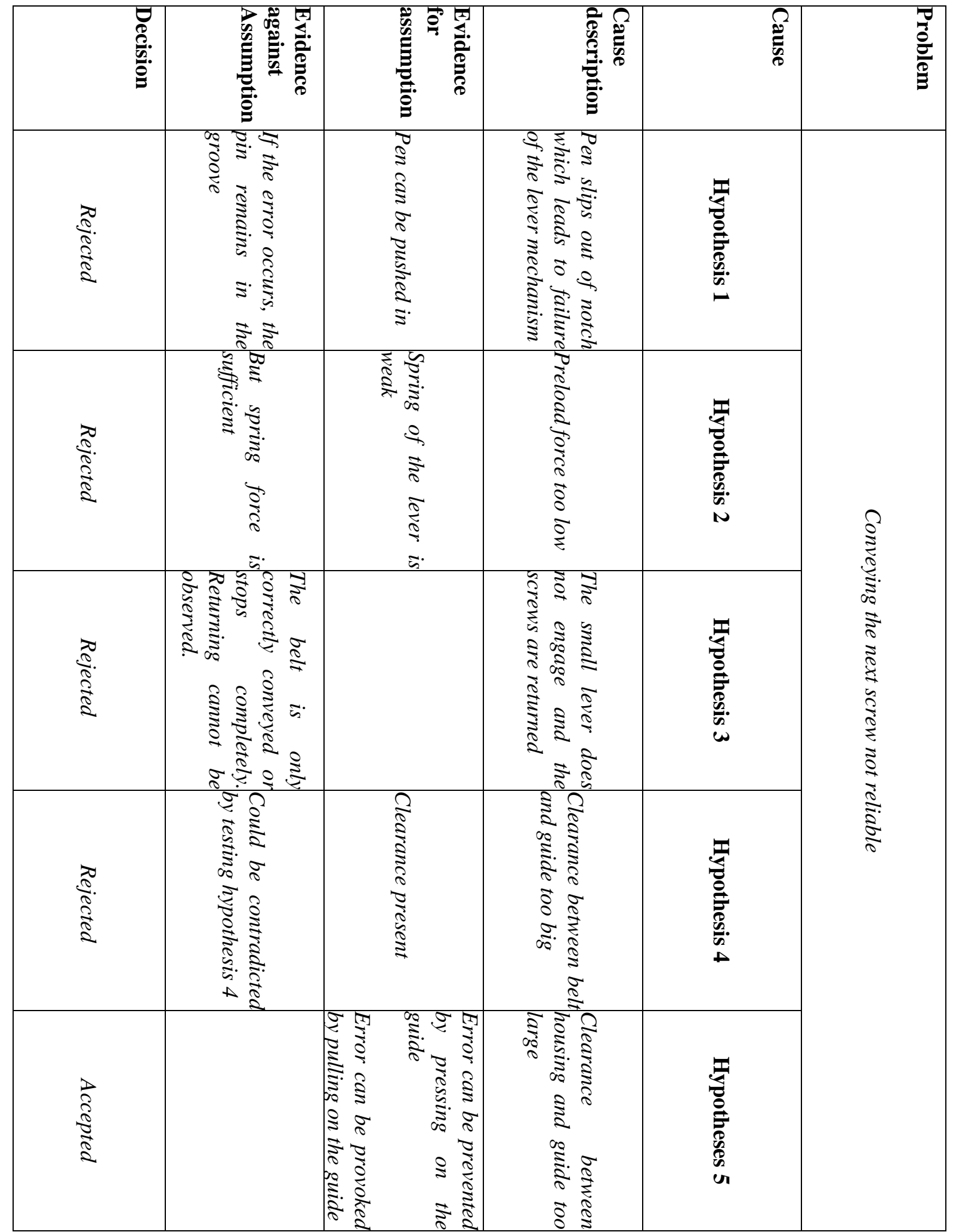

